RESEARCH ARTICLE

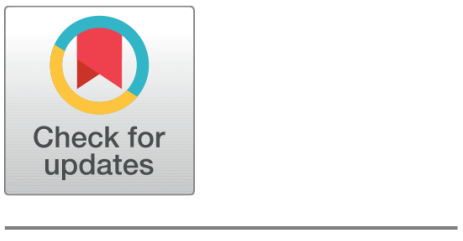

OPEN ACCESS

Received: 14.04.2021

Accepted: 29.04.2021

Published: 12.05 .2021

Citation: Paragas DS, Cruz KDC, Fiegalan ER (2021) Fermented Neem (Azadirachta indica) leaves-metal nanoparticles and their insecticidal properties against Bactrocera dorsalis (Hendel). Indian Journal of Science and Technology 14(17): 1338-1346. https://doi.org/ 10.17485/IJST/v14i17.631

*orresponding author.

danilaparagas1010@clsu.edu.ph

Funding: None

Competing Interests: None

Copyright: @ 2021 Paragas et al. This is an open access article distributed under the terms of the Creative Commons Attribution License, which permits unrestricted use, distribution, and reproduction in any medium, provided the original author and source are credited.

Published By Indian Society for Education and Environment (iSee)

ISSN

Print: 0974-6846

Electronic: 0974-5645

\section{Fermented Neem (Azadirachta indica) leaves-metal nanoparticles and their insecticidal properties against Bactrocera dorsalis (Hendel)}

\author{
Danila S Paragas ${ }^{1,2 *}$, Kathlia D C Cruz ${ }^{1}$, Elaida R Fiegalan ${ }^{3}$ \\ 1 School of Chemical, Biological and Materials Engineering and Sciences, Mapua University, \\ Intramuros, Manila, Philippines \\ 2 Department of Chemistry, College of Science, Central Luzon State University, Science City of \\ Muñoz, 3120, Nueva Ecija, Philippines \\ 3 Department of Crop Protection, College of Agriculture, Central Luzon State University, \\ Science City of Muñoz, 3120, Nueva Ecija, Philippines
}

\section{Abstract}

Objectives: To investigate the potential of nanoparticles synthesized from neem leaves in pest management for Bactrocera dorsalis. Methods: This study involves extracting the insecticidal properties of neem (Azadirachta indica) by fermenting air-dried leaves with rice wash. The crude fermented neem extract (FNE) was applied as a biopesticide against male $B$. dorsalis in an improvised olfactometer. The remaining crude extract was utilized in the green synthesis of silver nanoparticles (AgNP) and copper nanoparticles (CuNP). The insecticidal activities of FNE and the extracts with AgNP and CuNP were tested against B. dorsalis. Findings: The experimental treatments 100\% FNE and 20\% FNECuNP have the same effect as the positive control, causing the death of adult male fruit fly of $83.33 \%$ mortality rate after $24 \mathrm{~h}$. The treatment $20 \%$ FNE-AgNP showed a higher mortality rate, $100 \%$, after $24 \mathrm{~h}$. Likewise, the results of the larvicidal activities infer that the most effective treatment with a mortality rate of $100 \%$ after 24 -h exposure is the extract with silver nanoparticles. Moreover, the neem extract, FNE-AgNP, and FNE-CuNP exhibited ovicidal properties, suppressing the development of eggs into third instar larvae. Fermented neem extract can be used to formulate biopesticide enhanced with nanoparticles for controlling $B$. dorsalis. Novelty: This study showed that the fermented neem leaves extract using rice wash is effective in the green synthesis of silver and copper nanoparticles. The prepared biopesticide metal nanoparticles can be used in the management of $B$. dorsalis.

Keywords: silver nanoparticles; copper nanoparticles; insecticidal; Bactrocera dorsalis; Azadirachta indica 


\section{Introduction}

Fruit flies [Diptera: Tephritidae] are among the utmost economically significant pest species globally ${ }^{(1)}$. The genus Bactrocera is widely dispersed throughout tropical Asia, the South Pacific, Australia, and other countries, including parts of America and Oceana and the sub-Saharan countries. They are well-documented invaders because of their superior mobility, high reproductive rates, and extreme polyphagy levels. Bactrocera dorsalis is now the most invasive species in many typical host fruits and vegetables ${ }^{(2)}$.

Various technologies have been utilized for area-wide control of Bactrocera fruit flies and related species throughout Asia and the Pacific ${ }^{(3-6)}$. The male annihilation technique was the oldest but is now being modified with new lures, lure mixtures and dispenser formulations ${ }^{(3)}$.

The application of nanoparticles in agricultural productivity enhancement is currently an emerging technology ${ }^{(7-13)}$. The discovery of naturally occurring nanostructures in insects has led researchers to see nanotechnology's potential in pest management. Nanostructure components are present in the different parts of the insect's body, varying from $200-1000 \mathrm{~nm}^{(14)}$.

Neem (Azadirachta indica, A. Juss) tree has been famous in agriculture, industry, medicine, and the environment because of its numerous bioactive ingredients such as triterpenoids and steroids, non-terpenoids, and non-steroid constituents ${ }^{(15-17)}$, which are known to have anticancer ${ }^{(18)}$, fumigants for stored products ${ }^{(19)}$, medicinal ${ }^{(20)}$, insecticidal ${ }^{(21,22)}$ and larvicidal ${ }^{(22)}$ properties.

In this study, rice wash was used to ferment neem leaves to extract the insecticidal properties against $B$. dorsalis. The fermented extract was further used to prepare silver nanoparticles (AgNP) and copper nanoparticles (CuNP). These nanoparticles were applied in experiments against fruit fly. The plant natural products (PNPs) from neem leaves are extracted via fermentation using the microorganisms in the rice wash and the endophytic fungi inherent in the neem leaves. Endophytic fungi are beneficial as biocontrol agents in plant protection ${ }^{(23,24)}$.

\section{Materials and Methods}

\subsection{Chemicals}

The $\mathrm{CuSO}_{4} .5 \mathrm{H}_{2} \mathrm{O}, \mathrm{AgNO}_{3}, \mathrm{HCl}, \mathrm{NaOH}$ used in the preparation of nanoparticles, and the methyl eugenol used in trapping male fruit flies were of analytical grade.

\subsection{Plant Materials Collection and Preparation of Extract}

The neem branches were collected in the morning along the research avenue of Central Luzon State University, Science City of Muñoz, Nueva Ecija, Philippines. The branches were washed thoroughly with tap water and were air-dried for five days in the shade. The dry clean leaves were detached from the branches and were ground in a blender and stored in a tightly covered amber bottle at room temperature until used in the extraction process.

The fermentation method described by Sasidharan et al. ${ }^{(25)}$ was adopted in this experiment, except that the solvent used was rice wash.

\subsection{Optimization of parameters for the green synthesis of nanoparticles}

The procedure for the synthesis of AgNP and CuNP was described by Paragas, Cruz and Fiegalan (2020) ${ }^{(26)}$ with some modifications.

One millimolar of $\mathrm{AgNO}_{3}$ and $\mathrm{CuSO}_{4} \cdot 5 \mathrm{H}_{2} \mathrm{O}$ solutions was used to synthesize AgNPs and CuNPs by reduction reaction using the neem leaves extracts. A change in color from yellow to reddish-brown indicated the reduction of $\mathrm{Ag}^{+}$to $\mathrm{Ag}^{0}$ and $\mathrm{Cu}^{2+}$ to $\mathrm{Cu}^{0}$.

The synthesis of metal nanoparticles was optimized by varying the $\mathrm{pH}(5,7$, and 11$)$, temperature $\left(30^{\circ} \mathrm{C}, 56^{\circ} \mathrm{C}\right.$, and $\left.100^{\circ} \mathrm{C}\right)$, and volume ratio of aqueous neem leaves extract and working solutions $\left(\mathrm{AgNO}_{3}\right.$ and $\left.\mathrm{CuSO}_{4} \cdot 5 \mathrm{H}_{2} \mathrm{O}\right)$. The following volume ratios were used: 5:95, 10:90, 15:85, 20:80 and 25:75 of the neem extract to $\mathrm{AgNO}_{3}$ and 1:99, 5:95, 10:90, 15:85 and 20:80 to $\mathrm{CuSO}_{4} \cdot 5 \mathrm{H}_{2} \mathrm{O}$.

The optimum parameters for the synthesis of nanoparticles were evaluated by the absorbances showing a peak within the 400-440 nm range (for AgNP) and 500-600 nm (for CuNP). The nanoparticles were further analyzed through Fourier transform infrared (FTIR) spectroscopy, scanning electron microscopy, and zeta sizer. 


\subsection{Collection of Test Insects}

Male fruit flies were trapped inside a canister with cotton moistened with $0.5 \mathrm{~mL}$ methyl eugenol. The trap was placed under the mango or guava tree early in the morning. The canister was covered when there were enough fruit flies inside. Cotton dipped in sugar solution placed on the canister's net covering served as the fruit flies' food. One hour after introducing the sugar solution, the fruit flies were transferred to the olfactometer using a transparent tube.

B. dorsalis larvae were collected from infested guava fruits. The infested fruits which were picked on the ground were washed with tap water and were incubated in the laboratory for two days before collecting larvae. Those infested fruits taken from the tree were cleaned with tap water and incubated in the laboratory for five days. The larvae used in the larvicidal effect were second and third instar.

Guava fruits with bruises (pricked by female fruit flies) were picked from the guava tree. These were washed with tap water and rinsed with distilled water. The fruits were then placed in twelve (12) separate beakers covered with Petri dishes and incubated for one week.

\subsection{Mortality Test}

\subsubsection{Adulticidal Effect}

The neem-metal nanoparticles' (AgNPs and CuNPs) adulticidal activities were tested using the improvised olfactometer described in the earlier reports of Paragas, Cruz and Fiegalan ${ }^{(21,26)}$. The mortality rates were observed at 6, 12, and $24 \mathrm{~h}$.

Treatment applications were the following: Treatment 1 (Rice wash - RWa); Treatment 2 (100\% fermented rice wash neem extract - FNE); Treatment 3 (20\% FNE-AgNP); Treatment 4 (20\% FNE-CuNP); and Treatment 5 (control). Percent mortality was computed using that described by Abbot ${ }^{(27)}$.

\subsubsection{Larvicidal effect}

Larvicidal activity of the dilute fermented rice washes neem extracts (100\% FNE), and the 20\% FNE-AgNP and 20\% FNE-CuNP were tested to the second and third instar larvae of the fruit fly. The percentage mortality was recorded after 6,12 , and $24 \mathrm{~h}$.

\subsubsection{Ovicidal effect}

Ovicidal activity of the 100\% FNE, 20\% FNE-AgNP, and 20\% FNE-CuNP was also tested. Twelve ripe guava fruits of almost exact sizes infested with fruit flies were collected, washed with tap water, and rinsed with distilled water. These were placed in separate $250-\mathrm{mL}$ beakers and were added with $5 \mathrm{~mL}$ of the extracts. The fruits were rotated every day to ensure that the extract can penetrate the inside of the fruit. On the fifth day, larvae that come out of the solution and those inside the fruits were counted. Dead larvae were identified and counted as $1^{\text {st }}, 2^{\text {nd }}$, or $3^{\text {rd }}$ instar. The fruit was opened, and living larvae were picked using tweezers and placed in a petri dish. The larvae were likewise identified and counted.

\subsection{Statistical Analysis}

All the experiments were done in three replications. The percentage mortalities of adult fruit flies and larvicidal and ovicidal effects were evaluated using analysis of variance (ANOVA). Post hoc analysis using Duncan Multiple Range Test (DMRT) was done for significant variances $(\mathrm{a}=0.05)$. A PASW Statistics 18 software was used.

\section{Results and Discussion}

\subsection{Green Synthesis and Characterization of Metal Nanoparticles}

The resulting filtered neem leaves rice wash extract was brown. The crude extract changes in color when subjected to different $\mathrm{pH}$ during the optimization procedure for nanoparticle synthesis. At $\mathrm{pH}$, the extract was transparent (colorless), becoming yellow at $\mathrm{pH} 7$ and brownish at $\mathrm{pH} 11$. A change in color of the crude extracts was also observed visually as the concentration was increased. The higher the concentrations of the extracts, the darker the color. For temperature, the color of neem crude extract was light yellow at $30^{\circ} \mathrm{C}$ to orange-brown at $100^{\circ} \mathrm{C}$.

The effects of $\mathrm{pH}$, temperature and volume ratio (neem extract: metal solution) on the surface plasmon resonance of the FNE-CuNP and FNE-AgNP are analyzed using UV-Vis spectrophotometry. The optimum parameters for the preparation of $\mathrm{AgNP}$ and $\mathrm{CuNP}$ are $\mathrm{pH} 11,100^{\circ} \mathrm{C}$, and 20:80 volume ratio.

FTIR spectroscopy was used to study the interaction between the metabolites present in the plant extracts and the synthesized metal nanoparticles. Figure 1A is the FTIR spectroscopic spectra of the FNE, FNE-AgNP, and FNE-CuNP. The FTIR spectra 
of the FNE-AgNP and FNE-CuNP retain the FNE's characteristic peaks, but distinct peaks' intensities were changed. The slight changes in the peaks' intensities imply the possible adsorption of small amounts of the functional groups responsible for reducing metal ions, such as the hydroxyl group, to metal nanoparticles' surface ${ }^{(26)}$. A significant change in the intensities of $\mathrm{O}-\mathrm{H}$ stretching and $\mathrm{N}-\mathrm{H}$ vibrations are noticeable. These may be the functional groups responsible for reducing the silver and copper ions, serving as capping and stabilizing agents.

Figure $1 \mathrm{~B}$ shows the SEM images of FNE-AgNP and FNE-CuNP. The images show that AgNPs are smaller than CuNP. FNEAgNP has a mean radius of $16.92 \pm 6.12 \mathrm{~nm}$ while $238.20 \pm 20.19$ for the FNE-CuNP. The surface charge, as measured by the zeta potential using a zeta sizer, of the FNE-AgNP is $-21.72 \pm 0.09$ while FNE-CuNP is $-22.85 \pm 0.28$ (Table 1 ).
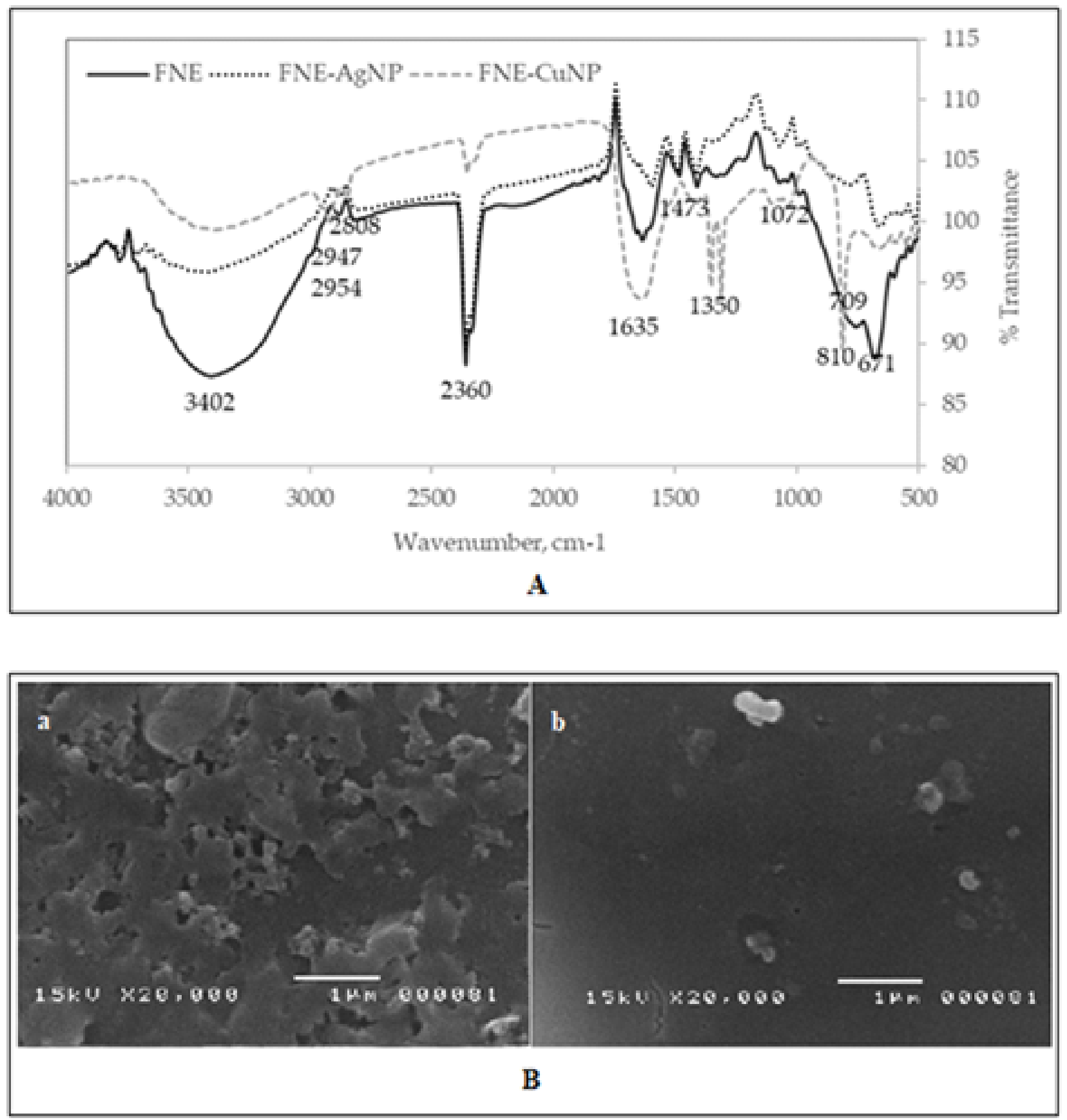

Fig 1. (A) FTIR spectra of the samples; (B) SEM image of (a) FNE-AgNP and (b) FNE-CuNP at $15 \mathrm{kV}$ and x 20,000 
Table 1. Some properties of the FNE-metal nanoparticles analyzed using a Zetasizer.

\begin{tabular}{lllllll}
\hline Nanoparticle & $\begin{array}{l}\text { Diffusion coefficient }\left(\mathrm{cm}^{2} / \mathrm{s}\right. \\
\left.\mathrm{x} 10^{-7}\right)\end{array}$ & $\begin{array}{l}\text { Zeta } \\
(\mathrm{mV})\end{array}$ & potential & $\begin{array}{l}\text { Mobility } \\
\mathrm{cm} / \mathrm{s} \mathrm{V})\end{array}$ & $\begin{array}{l}(\mu \mathrm{m} \\
(\mathrm{mS} / \mathrm{cm})\end{array}$ & $\begin{array}{l}\text { Conductivity } \\
(\mathrm{mean} \text { Radius }(\mathrm{nm})\end{array}$ \\
\hline FNE-AgNP & 8.3 & $-21.72 \pm 1.11$ & $-1.66 \pm 0.09$ & $0.27 \pm 0.04$ & $16.92 \pm 6.12$ \\
FNE-CuNP & 0.6 & $-22.85 \pm 0.28$ & $-1.75 \pm 0.03$ & $1.85 \pm 0.04$ & $238.20 \pm 20.19$ \\
\hline
\end{tabular}

\subsection{Mortality Test}

\subsubsection{Adulticidal Activity}

At 24-h exposure of the fruit flies to the various treatments, no fruit fly died in the rice wash. It was expected because the rice wash water has no substance that is toxic to the fruit flies. The highest mortality was registered in the FNE-AgNP (100\%) but is not significantly different from FNE, FNE-CuNP, and positive control (83.33\%). This result infers that the green synthesized $\mathrm{CuNP}$ is as effective as the FNE after $24 \mathrm{~h}$. The green synthesized AgNP, on the other hand, is even better than the FNE and the positive control. A study on the effect of nano-silica against Sitophilus oryzae has proven the entomotoxicity of nanoparticles. Its efficacy against the pest is higher than the bulk-sized silica with $90 \%$ mortality ${ }^{(28)}$. In this study, the nanoparticles' effect is evident, that a 20\% extract (from the 20:80 ratio in AgNP synthesis) is comparable with 100\% fermented neem leaves extract. This result implies that when the nanoparticles transport the active components such as the terpenoids, saponins, alkaloids in the neem extract, the higher is the effect on the fruit flies' mortality. These phytochemicals are responsible for reducing $\mathrm{Ag}^{+}$ into $\mathrm{Ag}^{0}$ and serving as the capping agent of the nanoparticles.

The AgNP or CuNP may block the input from phagostimulatory receptors ${ }^{(29)}$. Azadirachtin, a tetranortriterpenoid limonoid from the neem seeds and limonoid and sulfur-containing compounds in other parts of the plant, such as leaves and barks, affects the behavioral effects of insects through the chemoreceptor mechanism ${ }^{(30)}$. In the first few minutes after the extracts were introduced in the olfactometer, the fruit flies' initial response was avoidance. The fruit flies phagostimulants are widely distributed in the adult body, legs, wings, and the proboscis' labellum, the external gustatory centers. All these phagostimulants were detected and transcribed through the gustatory receptor neurons $(\mathrm{GRNs})^{(29)}$. Movement of the legs and spreading of the wings without body movement a few seconds after introducing the rice wash-neem extracts and nanoparticles in the olfactometer were observed. This behavior is manifested by the legs' movement and suppresses proboscis extension ${ }^{(31)}$. Effects on the proboscis by the various extracts are shown in Figure 2(a-c) and nanoparticles aggregated in the wings (Figure 2d).

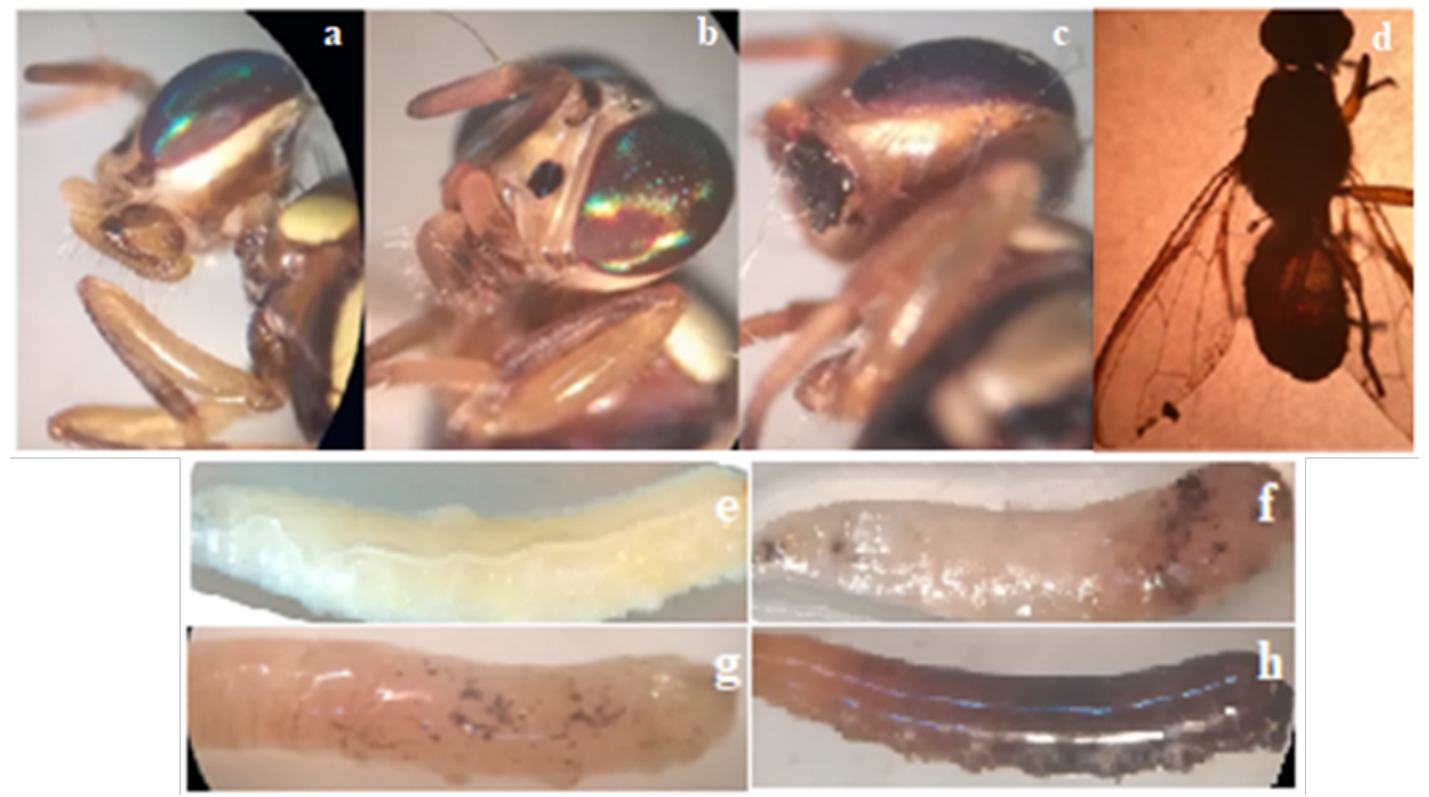

Fig 2. Effect of FNE (a), FNE-CuNP (b), and FNE-AgNP (c) on the proboscis of the fruit flies and presence of particles in the wings (suspected to be aggregated nanoparticles) in (d); typical $2^{\text {nd }}$ instar larva (e); effects of FNE (f), FNE-CuNP (g), and FNE-AgNP on $2^{\text {nd }}$ instar larvae of B. dorsalis. 
There are also instances where the flies immediately go near the extracts and ingest. According to Stocker, this phenomenon is where the fly detects the taste only after ingestion. Ingestion happens in the pharynx where the labral sense organ, the ventral and dorsal cibarial sense organs, and a central and dorsal row of fish-trap bristles detected the tastant ${ }^{(32)}$. Loss of fly mobility occurs when the flies' phagostimulant organs have ingested some of the extracts. GRNs transmit directly to the center of the brain, resulting in hyperactivity. Rattan ${ }^{(33)}$ reported that the hyperactivity was possibly due to neuroexcitation. The slow mobility could be due to energy depletion, neuromuscular fatigue, and neuroinhibition by possible oxygen deprivation and reduced respiratory capacity, leading to mortality. Armstrong et al. ${ }^{(34)}$ reported that AgNPs, inhibited membrane-bound copper-transporters, resulting in intracellular copper depletion and caused the reduction of tyrosine hydroxylase activity that reduces adult locomotive activity and reproductive development in Drosophila ${ }^{(35)}$.

The FNE-CuNP treatment showed anti-fruit fly activity of $83.33 \%$ mortality at $12 \mathrm{~h}$. Copper is regarded as essential to arthropods' physiological activity due to its role in hemocyanin ${ }^{(33)}$. This respiratory protein transports oxygen throughout the bodies of some invertebrates. The hemocyanin role may be the reason why FNE-CuNP has lower activity than FNE-AgNP. Moreover, the anti-fruit fly activity of CuNPs could be due to the $\mathrm{Cu}$ consumed by the fruit flies for the respiratory protein. Since the copper carries with it the phytochemicals from the neem that also inhibit proliferation and causes reduction rates of protein synthesis, accumulation of these phytochemicals finally leads to cell death. A lower than $100 \%$ mortality in the FNE$\mathrm{CuNP}$ at $24 \mathrm{~h}$ than FNE-AgNP can be attributed to copper's function in the fruit flies' respiratory protein and oxygen transport. The role of copper in the fruit fly, in this case, is dual. The FNE-AgNP's dual functions in the fruit flies' mortality are to inhibit membrane-bound copper transporters and reduce tyrosine hydroxylase activity ${ }^{(35)}$.

The other properties that can affect the activity of the FNE-AgNP and FNE-CuNP are the diffusion coefficient, surface charge, mobility, and conductivity, and mean radius of the nanoparticles. The FNE-AgNP has a higher diffusion coefficient than the FNE-CuNP. Tolaymat et al. ${ }^{(36)}$ mentioned that the mobility of silver nanoparticles is affected by the surface charge. Usually, silver nanoparticles have a negative surface charge in the $\mathrm{pH}$ range of 4-9. Electric fields affect wing movement, leading to agitation and changes in flies' brain chemistry ${ }^{(37)}$. In this study, upon placing the FNE-AgNP and FNE-CuNP on the olfactometer's upper portion, changes in the flies' behavior were observed. The fruit flies were attracted to the AgNPs and CuNPs with negative surface charges. As measured by the zeta potential using a zeta sizer, the surface charge of the FNE-AgNP is $-21.72 \pm 0.09$ while FNE-CuNP is $-22.85 \pm 0.28$ (Table 1 ). The difference in the fruit flies' mortality can also be due to the size of the nanoparticles. FNE-AgNP has a mean radius of $16.92 \pm 6.12 \mathrm{~nm}$ while $238.20 \pm 20.19$ for the FNE-CuNP.

\subsubsection{Larvicidal Activity}

The larvicidal effect of the extract is its ability to halt spontaneous parasite movement. Figure 2 confirms the effectiveness of the extracts with nanoparticles. Rice wash water has no larvicidal effect even at $24 \mathrm{~h}$ of exposure. FNE-AgNP showed the highest larvicidal activity (100\%), followed by FNE (91.67\%) and FNE-CuNP (75.00\%). Analysis of variance showed significant differences in the treatments. Post hoc analysis (DMRT) revealed that FNE-AgNP has the most significant larvicidal activity but is as effective as FNE. This result conforms with Santhoshkumar et al.s findings ${ }^{(38)}$. AgNP synthesized using methanol has higher activity than crude extracts.

Figure 2(f-h) demonstrate the effects of the FNE, FNE-AgNPs, and FNE-CuNPs on the larvae of $B$. dorsalis. Neem leaves extract affected the open circulatory system of the fly larva. The dark spots near the heart's posterior terminus are visible, which means that the hemolymph's circulation is disrupted. The effects of FNE-AgNP and FNE-CuNP on the dorsal vessels are shown in Figure 2(g) and (h). The posterior terminus of the larvae was the first affected. The darkening or formation of spots in the posterior terminus of the larva was prominent. FNE-AgNP has more considerable damage to the circulatory system than the FNE-CuNP.

Some larvae did not show the formation of dark spots. They continued to feed on the extracts and proceeded to the next larval stage, the third instar larva. Some pupated but with abnormalities (Figure 3 ) like non-retraction of the anterior end, blackish anterior, semi-transparent puparium, and no flies came out of the puparium after few days. The abnormal and empty puparium means that the neem extract and the nanoparticles could have functioned as antiecdysones that prevented the synthesis and release of natural juvenile hormones or speed up the destruction of these hormones leading to the premature metamorphosis of the larvae. These events mainly arise from the presence of an exoskeleton, which is cast off and re-formed at each ecdysis ${ }^{(39)}$. 


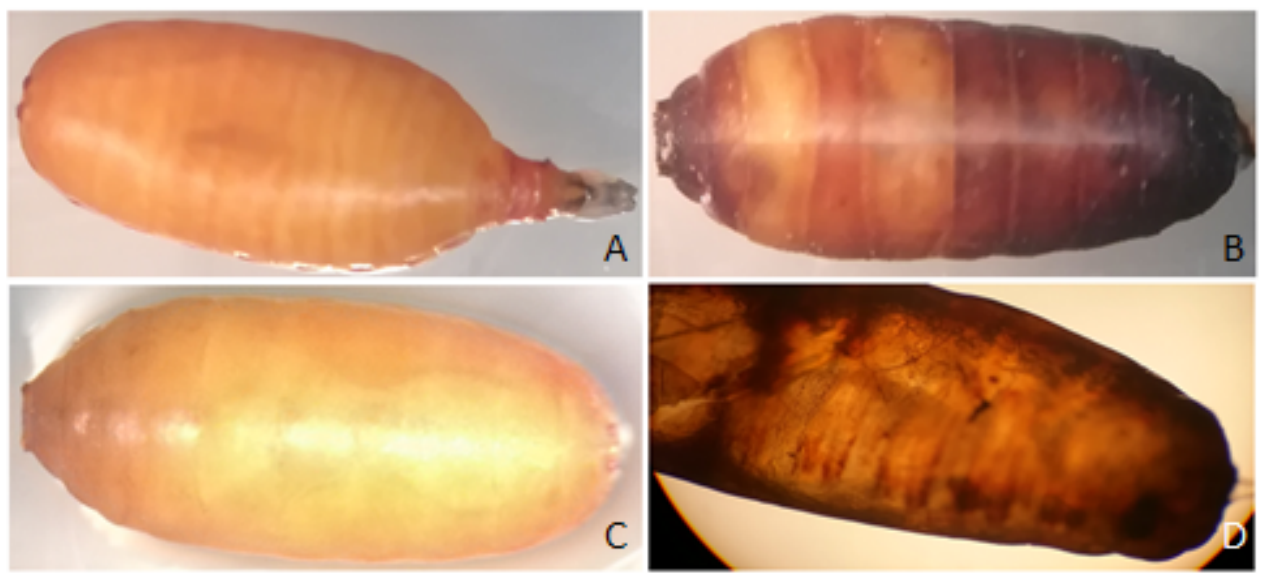

Fig 3. Some abnormalities observed to larvae affected by neem extracts and nanoparticles: A - non-retraction of the anterior end; B - blackish anterior end; $\mathrm{C}$ - semi-transparent puparium; and D - empty puparium.

\subsubsection{Ovicidal Activity}

The treatments' ovicidal effect was determined using the guava fruits infested with fruit flies incubated in beakers with the treatments for five (5) days. At the end of 5 days, the fruits were opened, and the larvae inside and outside the fruits were counted. Thirty-seven (37) larvae were developed from the eggs (Table 2 ). The FNE and FNE-AgNP have a mean value of 4.50 larvae, while and FNE-CuNP has a higher mean number of larvae (5.67) from the eggs.

Table 2. The emergence of larvae in the fruits exposed with the treatments for 5 days.

\begin{tabular}{lllll}
\hline \multirow{2}{*}{ Treatment } & \multicolumn{2}{l}{ Mean Number of Living Larvae (4 replicates) } & & Mean \\
\cline { 2 - 5 } & 1st Instarns & 2nd Instar & 3rd Instar & 10.5 \\
\hline Control (no treatment) & 10 & 16.5 & 0 & $4.00 \mathrm{a}$ \\
FNE & 0 & 4.5 & 0 & $4.50 \mathrm{~b}$ \\
FNE-AgNP & 3.25 & 1.25 & 0 & $5.67 \mathrm{~b}$ \\
FNE-CuNP & 5.67 & 0 & 0 & \\
\hline
\end{tabular}

${ }^{\star}$ Means with the same letter superscripts in a column are not significantly different at the $5 \%$ level by DMRT.

This result proves that the fermented neem extracts with AgNP and CuNP can suppress eggs' development into larvae. Silva et al. ${ }^{(40)}$ reported earlier that neem extracts using different parts (leaves, barks, and seed kernels) showed sub lethal effects against Ceratitis capitata, Mediterranean fruit fly adults. In this experiment the components in the extracts or maybe the AgNP and the CuNP capping the bioactive agents could penetrate to the inside of the fruit where the fly oviposited the eggs.

\section{Conclusion}

This study concludes that the fermented neem leaves using rice wash could be used in the green synthesis of AgNP and CuNP. The neem extract with nanoparticles exhibited mortality against male adult fruit fly, second instar larvae and eggs of Bactrocera dorsalis. Fermented neem extract can be used to formulate biopesticide enhanced with nanoparticles for pest management of B. dorsalis.

\section{Acknowledgement}

The researchers acknowledge the research fund from the Faculty Development Program of the Central Luzon State University, Science City of Muñoz, Nueva Ecija, Philippines. 


\section{References}

1) Vargas R, Piñero J, Leblanc L. An Overview of Pest Species of Bactrocera Fruit Flies (Diptera: Tephritidae) and the Integration of Biopesticides with Other Biological Approaches for Their Management with a Focus on the Pacific Region. Insects. 2015;6(2):297-318. Available from: https: //dx.doi.org/10.3390/insects6020297.

2) Roger IV, Luc L, Rudolph P, Avi E. Impact of introduction of Bactrocera dorsalis (Diptera: Tephritidae) and classical biological control releases of Fopiusarisanus (Hymenoptera: Braconidae) on economically important fruit flies in French Polynesia. Journal of Economic Entomology. 2007;100(3):670679. Available from: https://doi.org/10.1093/jee/100.3.670.

3) I VR, L L, C PJ, M HK. Male Annihilation, Past, Present, and Future. In: T S, N E, E J, J RF, R V, editors. Trapping and the detection, control, and regulation of tephritid fruit flies. Dordrecht. Springer. 2014;p. 493-511. Available from: https://doi.org/10.1007/978-94-017-9193-9_14.

4) Verghese A, Tandon PL, Stonehouse JM. Economic evaluation of the integrated management of the oriental fruit fly Bactrocera dorsalis (Diptera: Tephritidae) in mango in India. Crop Protection. 2004;23(1):61-63. Available from: https://dx.doi.org/10.1016/s0261-2194(03)00087-5.

5) Piñero JC, Mau RFL, Vargas RI. Managing Oriental Fruit Fly (Diptera: Tephritidae), With Spinosad-Based Protein Bait Sprays and Sanitation in Papaya Orchards in Hawaii. Journal of Economic Entomology. 2009;102(3):1123-1132. Available from: https://dx.doi.org/10.1603/029.102.0334.

6) John DS, Roger IV, Steven KS, Jo FA, Randall ST, Luc L, et al. Simulated field applications of insecticide soil drenches for control of tephritid fruit flies. Biopesticides International. 2014;10(2):136-142. Available from: https://www.researchgate.net/publication/274779864.

7) Pradhan S, Mailapalli DR. Nanopesticides for Pest Control. In: and others, editor. Sustainable Agriculture Reviews;vol. 40. Springer. ;p. 43-74. Available from: https://doi.org/10.1007/978-3-030-33281-5_2.

8) Anandhi S, Saminathan VR, Yasotha P, Saravanan PT, Venugopal R. Nano-pesticides in pest management. Journal of Entomology and Zoology Studies. 2020;8(4):685-690.

9) Narasimhamurthy K, Soumya K, Undayashankar CA, Srinivas C, Roshan A. Ramachandrappa Nirianjana Siddapura. Nanofertilizers and nanopesticides: Recent trends, future prospects in agriculture. Advances in Nano-Fertilizers and Nano-Pesticides in Agriculture. 2021;p. 281-330. Available from: https://doi.org/10.1016/B978-0-12-820092-6.00012-4.

10) Sabour MM. Entomotoxicity Assay of Nanoparticle 4-[silica gel Cab-O-Sil-750, Silica Gel Cab-O-Sil-500] against Sitophilus oryzae under Laboratory and Stored Conditions in Egypt. Science Research Reports. 2013;1(2):67-74.

11) Arnab D, Rituparna B, Ajeet K, Subho M. Targeted delivery of pesticides using biodegradable polymeric nanoparticles. New Delhi; India. Springer. 2014. doi:10.1007/978-81-322-1689-6.

12) Pereira AES, Grillo R, Mello NFS, Rosa AH, Fraceto LF. Application of poly(epsilon-caprolactone) nanoparticles containing atrazine herbicide as an alternative technique to control weeds and reduce damage to the environment. Journal of Hazardous Materials. 2014;268:207-215. Available from: https://dx.doi.org/10.1016/j.jhazmat.2014.01.025.

13) Simonian AL, Good TA, Wang SS, Wild JR. Nanoparticle-based optical biosensors for the direct detection of organophosphate chemical warfare agents and pesticides. Analytica Chimica Acta. 2005;534(1):69-77. Available from: https://dx.doi.org/10.1016/j.aca.2004.06.056.

14) Bhattacharyya A, Bhaumik A, Rani PU, Mandal S, Epidi TT. Nano-particles-A recent approach to insect pest control. African Journal of Biotechnology. 2010;9(24):3489-3493. Available from: https://www.ajol.info/index.php/ajb/article/view/82345.

15) Ugochukwu BA. Medicinal properties of fractionated acetone/water neem Azadirachta indica leaf extract from Nigeria: a review. Nigerian Journal of Physiological Sciences. 2010;24(2):157-159. Available from: https://dx.doi.org/10.4314/njps.v24i2.52926.

16) Bigoniya P, Singh CS, Srivastava B. Pharmacognostical and physico-chemical standardization of Syzygium cumini and Azadirachta indica seed. Asian Pacific Journal of Tropical Biomedicine. 2012;2(1):S290-S295. Available from: https://dx.doi.org/10.1016/s2221-1691(12)60176-2.

17) Babatunde DE, Otusemade GO, Efeovbokhan VE, Ojewumi ME, Bolade OP, Owoeye TF. Chemical composition of steam and solvent crude oil extracts from Azadirachta indica leaves. Chemical Data Collections. 2019;20:100208. Available from: https://dx.doi.org/10.1016/j.cdc.2019.100208.

18) Paul R, Prasad M, Sah NK. Anticancer biology ofAzadirachta indicaL (neem): A mini review. Cancer Biology \& Therapy. 2011;12(6):467-476. Available from: https://dx.doi.org/10.4161/cbt.12.6.16850.

19) Koul O. Biological Activity of Volatile Di-n-Propyl Disulfide from Seeds of Neem, Azadirachta indica (Meliaceae), to Two Species of Stored Grain Pests, Sitophilus oryzae (L.) and Tribolium castaneum (Herbst). Journal of Economic Entomology. 2004;97(3):1142-1147. Available from: https: //dx.doi.org/10.1093/jee/97.3.1142.

20) Kausik B, Ishita C, Ranajit KB, Uday B. Biological activities and medicinal properties of neem (Azadirachta indica). Current Science. 2002;10:1336-1345. Available from: https://www.jstor.org/stable/24106000.

21) Paragas DS, Cruz KDC, Fiegalan ER. Screening of plants for insecticidal activities against oriental fruit fly Bactrocera dorsalis (Hendel) for pest management of mango Mangifera indica. Malaysian Journal of Analytical Sciences. 2020;24(4):615-644. Available from: https://mjas.analis.com.my/ mjas/v24_n4/pdf/Paragas_24_4_15.pdf.

22) Shanmugasundaram R, Jeyalakshmi T, Dutt MS, Murthy PB. Larvicidal activity of neem and karanja oil cakes against mosquito vectors, Culex quinquefasciatus (Say). Journal of Environmental Biology. 2007;29(1):43-45. Available from: https://pubmed.ncbi.nlm.nih.gov/18831329/.

23) Rajagopal K, Suryanarayanan TS. Isolation of endophytic fungi from leaves of neem (Azadirachta indica A. Juss.). Current Science. 2000;78(11):1375-1378. Available from: https://www.jstor.org/stable/24104047.

24) Verma VC, Gond SK, Kumar A, Kharwar RN, Strobel G. The Endophytic Mycoflora of Bark, Leaf, and Stem Tissues of Azadirachta indica A. Juss (Neem) from Varanasi (India). Microbial Ecology. 2007;54(1):119-125. Available from: https://dx.doi.org/10.1007/s00248-006-9179-9.

25) Sasidharan S, Chen Y, Saravanan D, Sundram KM, Latha LY. Extraction, Isolation And Characterization Of Bioactive Compounds From Plants' Extracts. African Journal of Traditional, Complementary and Alternative Medicines. 2011;8(1):1-10. Available from: https://dx.doi.org/10.4314/ajtcam.v8i1.60483.

26) Paragas DS, Cruz KDC, Fiegalan ER. Green synthesized copper nanoparticles from Blumea balsamifera Linn. leaves and its biocidal activities against Bactrocera dorsalis (Hendel). Malaysian Journal of Analytical Sciences. 2009;24(3):436-484. Available from: https://mjas.analis.com.my/mjas/v24_n3/ pdf/Paragas_24_3_14.pdf.

27) Abbott WS. A method of computing the effectiveness of an insecticide. Journal of the American Mosquito Control Association. 1987;3(2):302-303. Available from: http://pascal-francis.inist.fr/vibad/index.php?action=getRecordDetail\&idt=8376553.

28) Debnath N, Das S, Seth D, Chandra R, Bhattacharya SC, Goswami A. Entomotoxic effect of silica nanoparticles against Sitophilus oryzae (L.). Journal of Pest Science. 2011;84(1):99-105. Available from: https://dx.doi.org/10.1007/s10340-010-0332-3.

29) Depetris-Chauvin A, Galagovsky D, Grosjean Y. Chemicals and chemoreceptors: ecologically relevant signals driving behavior in Drosophila. Frontiers in Ecology and Evolution. 2015;3:41. Available from: https://dx.doi.org/10.3389/fevo.2015.00041. 
30) (Luntz) AJM, Nisbet AJ. Azadirachtin from the neem tree Azadirachta indica: its action against insects. Anais da Sociedade Entomológica do Brasil. 2000;29(4):615-632. Available from: https://dx.doi.org/10.1590/s0301-80592000000400001. doi:10.1590/s0301-80592000000400001.

31) Kim H, Kirkhart C, Scott K. Long-range projection neurons in the taste circuit of Drosophila. eLife. 2017;6:1-24. Available from: https://dx.doi.org/10. 7554/elife.23386.

32) Stocker RF. The Olfactory Pathway of Adult and Larval Drosophila. Annals of the New York Academy of Sciences. 2009;1170(1):482-486. Available from: https://dx.doi.org/10.1111/j.1749-6632.2009.03896.x.

33) Rattan RS. Mechanism of action of insecticidal secondary metabolites of plant origin. Crop Protection. 2010;29(9):913-920. Available from: https: //dx.doi.org/10.1016/j.cropro.2010.05.008.

34) Armstrong N, Ramamoorthy M, Lyon D, Jones K, Duttaroy A. Mechanism of Silver Nanoparticles Action on Insect Pigmentation Reveals Intervention of Copper Homeostasis. PLoS ONE. 2013;8(1):e53186. Available from: https://dx.doi.org/10.1371/journal.pone.0053186.

35) Robert GP, Aseel R, Thomas S, Tim T, Ralph H. Effects of tyrosine hydroxylase mutants on locomotor activity in Drosophila: a study in functional genomics. Behavior Genetics. 2002;32(2):89-94. Available from: https://doi.org/10.1023/A:1015279221600.

36) Tolaymat TM, Badawy AME, Genaidy A, Scheckel KG, Luxton TP, Suidan M. An evidence-based environmental perspective of manufactured silver nanoparticle in syntheses and applications: A systematic review and critical appraisal of peer-reviewed scientific papers. Science of The Total Environment. 2010;408(5):999-1006. Available from: https://dx.doi.org/10.1016/j.scitotenv.2009.11.003.

37) Mesfer SAG. The effect of static electric fields on Drosophila behaviour . 2012. Available from: https://eprints.soton.ac.uk/342464/1/Binder1.pdf.

38) Thirunavukkarasu S, Rahuman A, Abdul R, Govindasamy M, Sampath B, Asokan J, et al. Synthesis of silver nanoparticles using Nelumbo nucifera leaf extract and its larvicidal activity against malaria and filariasis vectors. Parasitology research. 2011;108(3):693-702. Available from: 10.1007/s00436-0102115-4.

39) Hassall KA. The Biochemistry and Uses of Pesticides: Structure, Metabolism, Mode of Action, and Uses in Crop Protection. and others, editor;VCH Publishers. 1990. Available from: https://www.academia.edu/25755456/The_biochemistry_and_uses_of_pesticides_structure_metabolism_mode_of_ action_and_uses_in_crop_protection.

40) Silva MA, Bezerra-Silva GCD, Vendramim JD, Mastrangelo T. Sublethal effect of neem extract on mediterranean fruit fly adults. Revista Brasileira de Fruticultura. 2013;35(1):93-101. Available from: https://dx.doi.org/10.1590/s0100-29452013000100012. 